\title{
Ultrasound and management strategies in chronic pain
}

\author{
Hanik Badriyah Hidayati, MD, Neurologist, Consultant of Pain, PhD \\ Department of Neurology, Medical Faculty of Airlangga University of Airlangga University - Dr. Soetomo General Hospital, \\ Surabaya, East Java, Indonesia.
}

Correspondence: Hanik Badriyah Hidayati, Department of Neurology, Medical Faculty of Airlangga University - Dr. Soetomo General Hospital, Mayjend. Prof. Dr. Moestopo Street, Number: 6-8, Surabaya, East Java, Indonesia, 60286; E-mail: hanikhidayati@yahoo.com

\begin{abstract}
Pain is the most common reason for seeking medical advice. Chronic pain remains a significant major problem. Imaging techniques are essential tools in interventional pain management (IPM). Ultrasound (US) has been comparatively a recent technology and its wider use has and an increased interest in imaging in IPM. This editorial briefly introduces the application of the US in chronic pain management strategies, and highlights its application, benefits, evidence and limitations in IPM.

Keywords: Chronic pain; Interventional pain management; Ultrasound; Imaging

Citation: Hidayati HB. Ultrasound and management strategies in chronic pain. Anaesth. pain intensive care 2020;24(6):579-581.

Received: 15 September 2020, Reviewed: 26 September 2020, Accepted: 15 November 2020
\end{abstract}

\section{Introduction}

Pain, according to the latest definition by International Association for the Study of Pain (IASP) has been described as "An unpleasant sensory and emotional experience associated with, or resembling that associated with, actual or potential tissue damage," and is expanded upon by the addition of six key Notes and the etymology of the word pain for further valuable context. ${ }^{1,2}$ Pain can be experienced as an acute or chronic pain. ${ }^{2}$

Chronic pain has been defined by some authors as a persistent pain for 3-6 months. ${ }^{1}$ It remains a big problem with a significant health, social, and financial burden on the society. ${ }^{2,3}$ It is frequently accompanied by sleep, mood, and metabolic dysfunction and significantly affects the quality of life (QoL). ${ }^{3}$ Chronic pain can be a leading cause of loss of productivity, reduced work performance, and absenteeism from work. $^{2}$ The latest report infers that chronic pain affects approximately $20 \%$ of the world's population. It can occur at any age and the numbers of chronic pain continuously increase due to the ever-increasing number of aging population. ${ }^{3}$
In the recent past, ultrasound (US) has gained much popularity, and its application in interventional procedures in chronic pain management is very interesting. 5,6 It has multiple advantages over the radiographic imaging when used for interventional pain management (IPM). The use of image guidance in IPM has become a necessity, ${ }^{5}$ and US can play a significant role in the evaluation of the musculoskeletal system and IPM. ${ }^{7}$

\section{Ultrasound Application in IPM}

US is used for clinical application of musculoskeletal system, either diagnostic and therapeutic purpose. ${ }^{7}$ It can be applied in several areas of musculoskeletal system from head to toe, including head; cervical, thoracic, lumbar and sacral spine; and various joints. ${ }^{8}$

Possible applications of US blocks are nerve blocks, whether superficial or deep, zygapophyseal (facet) joint block, lumbar facet blocks, painful stump neuromas, and sympathetic chain and/or ganglia block..$^{5,7-10}$ US is also used for intra-articular injections of lumbar zygapophyseal joints, acromioclavicular joints, glenohumeral joints, sacroiliact joints, lower limb joints. Possible 
applications of US injections are muscle injections of pelvis muscle, piriformis muscle and injections of tender points. US is now routinely used for epidural injection at various levels, subacromion sub deltoid (SASD) bursa, and supraspinatus tendon. ${ }^{5,7-10}$

\subsection{The Benefits of US in IPM}

Imaging techniques are important tools in IPM. ${ }^{7}$ Its use offers many benefits in IPM; it allows IPM to be performed at the bedside in the wards or even intensive care units, and allows visualization and good resolution of muscle, vessels, nerves and connective tissues. It allows visualization of the target structure, needle movement and the spread of the solution injected continuously in a direct and real-time fashion. Thus, it has been claimed to reduce the therapeutic doses to be used, serves selective blocks with higher accuracy, due to its visibility of the target structure and local anaesthetic spread.

US guided injections are more effective than landmark guided injections, ${ }^{5}$ and are safer to the operator and the subject as there is no radiation exposure, which is a major hazard of using fluoroscopes or CT scans for IPM. Furthermore, there is no requirement of a separate area to perform the block when using US, and it is the only imaging-guidance equipment appropriate for use during pregnancy.

The development of newer hand held US probes, which can be used with mobile phone sets or computer tabs have revolutionized its use by making it as a portable and low-cost tool. ${ }^{11}$

\subsection{The Evidence of US in IPM}

The quality of scientific evidence of the benefits of US has been highly variable, with very few randomized controlled trials (RCT) involving patients published so far; a large part of the supporting evidence includes published case reports and studies related to the practicability and performance of the techniques. ${ }^{5}$ But there is a rapidly increasing interest in IPM, and with it in US as evidenced by the growing number of publications. ${ }^{6}$ Samer N. Narouze presented the evidence for US-guided procedures in some cases and categorized the cases into two main areas of interest: US-guided cervical and US-guided lumbar spine injections, as follows: ${ }^{8}$

\subsubsection{US-Guided Cervical Spine Injections}

For cervical selective nerve root blocks no recommendations can be given at this time if US is superior to fluoroscopy, because only limited data exist (level III). Also, for US-guided cervical facet intraarticular injections, only limited data based upon cadaveric experimental studies is available, so no recommendations can be made. There were few reports suggesting that US guidance has some advantages over fluoroscopy-guided injections, especially in stellate ganglion block (SGB) and cervical nerve root blocks, but there are no RCTdriven data to support this. ${ }^{6}$

\subsubsection{US-Guided Lumbar Spine Injection}

US cannot be recommended to be the solo imaging technique for lumbar nerve root blocks, especially in obese patients (level III-IV). However, for lumbar facet joint injections, US is superior to CT-guided injections in regard to radiation exposure and time to perform the procedure (1 small RCT, level Ib). No recommendations can be given regarding its superiority to fluoroscopy because no data exist. Similarly, no recommendations can be given if US is superior to fluoroscopy for lumbar selective nerve root block, as no clinical data exist. ${ }^{6}$

\section{Limitation of Ultrasound in IPM}

The use of US has limitations in some particular situations, and these may be related to patient factors, technical factors or operator factors.

\subsection{Patient Factors}

Patient factors include degenerative changes, abnormal anatomy, body habitus and obesity.,9 Obesity-related pain is related to physical disability due to obesity and metabolic dysregulation. ${ }^{3}$ The image quality in obese patients deteriorates in deeper planes. ${ }^{9}$

\subsection{Technical Factors}

Technical factors include a reduction in image resolution with depth, reduced needle visibility with a steep angle of insertion, and the bone shadowing or acoustic shadow artefact produced by bone. Bone shadowing and poor image resolution may diminish the visualization of the needle, identification of 
injectate spread, and also the ability to visualize intravascular injection, limiting the use of US in certain procedures. ${ }^{5}$

In several cases of routine clinical practice US cannot replace fluoroscopy or CT, for example: there is no way to use US guided injection to perform effectively transforaminal steroid injection or disc stimulation (discography). ${ }^{7}$ The use of US as the sole imaging modality for transforaminal epidural is not currently supported. ${ }^{5}$ Due to acoustic impedance mismatches of tissue interfaces, US cannot penetrate air or bone. Furthermore, it is not possible generally to see through structures containing air (e.g., lung) or through large bones. ${ }^{9}$ In this case and other applications, fluoroscopy has still no competition. ${ }^{7}$

\subsection{Operator Factors}

Operator factors include technical skill and experience. Only trained physicians with sufficient practice under supervision of an experienced sonographer should use it, until expertise with the method is gained. $^{7}$ Nerve sonography requires excellent anatomical knowledge and experience. The identification of the nerves is frequently difficult. Reading a literature review or a book chapter are not enough to learn US guided pain procedures. $^{7}$ It requires considerable effort and training to learn and implement new skills of US guided pain procedures. ${ }^{5}$

\section{Conclusion}

US has gained considerable popularity in chronic pain management. It is a valuable tool and promising imaging technique in chronic pain management, helping to target_injections, guiding the needle and confirming the spread of injectate_around the target. ${ }^{6,7}$ US has a potential benefit in interventional pain management, but also has some limitations. Further clinical trials to investigate efficacy and safety of US guided pain procedures is still needed.

\section{Conflict of interest}

The author declare has no conflict of interest.

\section{Acknowledgement}

The author is thankful to David Nugraha for helping in hyperlinking the references and Azham
Purwandono, MD, neurologist for checking plagiarism.

\section{References}

1. ASP Announces Revised Definition of Pain. Jul 16, 2020. Available at: https://www.iasppain.org/PublicationsNews/NewsDetail.aspx? ItemNum ber=10475\#: :text=The $\% 20$ definition $\% 20$ is $\% 3$ A $\% 20 \%$ E2\%80\%9CAn\%20unpleasant,pain \%20for $\% 20$ further $\% 20$ valuable $\% 20$ context. (Accessed on 30 November 2020).

2. Dureja GP, lyer RN, Das G, Ahdal J, Narang P. Evidence and consensus recommendations for the pharmacological management of pain in India. J Pain Res. 2017;10:709-736. [PubMed] [Free full text] DOI: $10.2147 / J P R . S 128655$

3. Bonakdar RA. Integrative Pain Management. Med Clin NA. 2017;101(5):987-1004. [PubMed] DOI: 10.1016/j.mcna.2017.04.012

4. Simpson G, Nicholls B. Use of ultrasound in chronic pain medicine. Part 1: Neuraxial and sympathetic blocks. Contin Educ Anaesthesia, Crit Care Pain. 2013;13(5):145-151. [Free full text] DOI: 10.1093/bjaceaccp/mkt013

5. Narouze SN. Ultrasound-Guided Interventional Procedures in Pain Management. Reg Anesth Pain Med. 2010;35(2 Suppl):S55-58. [PubMed] DOI: $10.1097 / A A P .0 b 013 e 3181 \mathrm{~d} 24658$

6. Eichenberger $U$, Greher M, Curatolo M. Ultrasound in interventional pain management. Tech Reg Anesth Pain Manag. 2004;8(4):171-178. [Free full text]

7. Peng $P$, Finlayson $\mathrm{R}$, Lee $\mathrm{SH}$, Bhatia $\mathrm{A}$, editors. Ultrasound for Interventional Pain Management. Ultrasound for Interventional Pain Management. Canada: Springer; 2020. p.1-362.

8. Sandhu NS. Ultrasound imaging in anesthesia: an overview of vascular access and peripheral nerve blocks. 2007;197-209. Seminar in Aneasthesia, Perioperative Medicine and Pain. DOI: 10.1053/j.sane.2007.10.003

9. Jacobson JA. Fundamentals of Musculoskeletal Ultrasound. 2nd Editio. Elsevier Saunders; 2013. p.492.

10. Korbe S, Udoji EN, Ness TJ, Udoji MA. Ultrasoundguided interventional procedures for chronic pain management. Pain Manag. 2015;5(6):465-482. [PubMed] [Free full text] DOI: 10.2217/pmt.15.46 